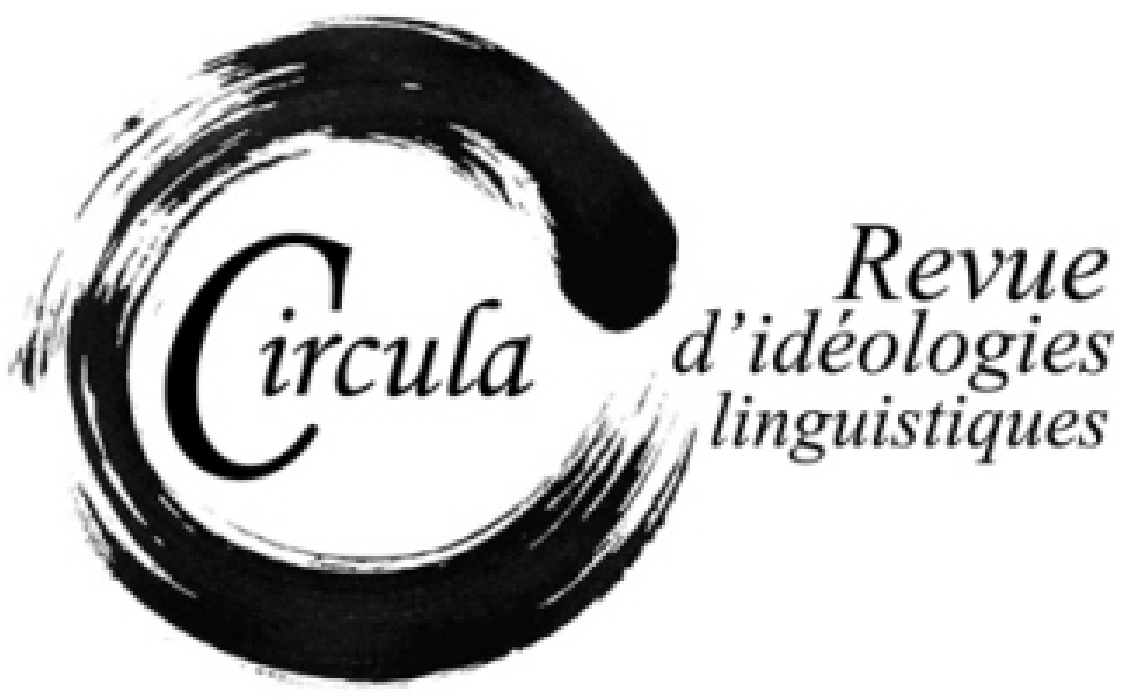

TITRE: S'OUVRIR AUX SUGGESTIONS DES LOCUTEURS POUR AMÉNAGER LA TERMINOLOGIE AU QUÉBEC : LE CAS DU PROJET COLLABORATIF «EN BONS TERMES»

Auteure: Elizabeth C. Saint, Université d'Ottawa

Revue: Circula, numéro 9 : A-T-on encore PeUR des anglicismes? Perception actuelle des anglicismes au QuébeC ET DANS L'ESPACE FRANCOPHONE

PAGES: $29-57$

ISSN: 2369-6761

DiReCTRICES: MiREILlE ElChaCAR ET NADINE VINCENT

URI: HTTP://HDL.HANDLE.NET/11143/16046

DOI: HTTPS://DOI.ORG/10.17118/11143/16046 


\title{
S'ouvrir aux suggestions des locuteurs pour aménager la terminologie au Québec : le cas du projet collaboratif « En bons termes »
}

\author{
Elizabeth C. Saint, Université d'Ottawa \\ esain104@uottawa.ca
}

Résumé : Cet article, ancré dans les recherches de l'implantation terminologique, présente les données du projet «En bons termes », qui a fait collaborer pendant quatre mois sur un forum en ligne divers acteurs de l'aménagement linguistique et terminologique canadien, dont l'Office québécois de la langue française (OQLF) et un groupe de locuteurs, dans le but de trouver des solutions terminologiques à des concepts sans dénomination en français. En nous concentrant sur les termes qui apparaissent sur les 11 fiches parues dans le Grand dictionnaire terminologique (GDT) à l'issue de ce projet, nous montrons comment l'OQLF a accueilli les suggestions des locuteurs, ainsi que la place et le statut d'acceptabilité qu'il leur a accordés dans les fiches. Les résultats montrent que l'organisme a fait preuve d'une réelle ouverture aux contributions des internautes et que celle-ci s'inscrit dans une attitude socioterminologique globale qui a pour objectif l'implantation réussie des termes dans l'usage.

Mots-clés : aménagement terminologique ; externalisation ouverte; projet collaboratif «En bons termes »; néologie aménagiste ; Office québécois de la langue française (OQLF)

Abstract: This article, framed within studies on term implantation, presents the results of the online collaborative project En bons termes between a group of speakers and various actors of language and terminology planning in Canada, including the Office québécois de la langue française (OQLF). The objective of the collaboration was to find suitable terminological solutions to name new concepts in French. With a focus on the terms that were published on 11 term records in the OQLF's termbank, Le Grand dictionnaire terminologique, we analyse how the OQLF integrated speakers' suggestions, including the space and acceptability status that it gave them in the records. Conclusions show that the OQLF was open to speakers' contributions, as per the general socioterminological orientation it adopted over the last couple of decades to ensure the successful implantation of its terminology.

Keywords: terminology planning; online collaboration En bons termes; crowdsourcing; planned neology; Office québécois de la langue française (OQLF) 


\section{Introduction}

En janvier 2017, l'Office québécois de la langue française (désormais, l'Office), adoptait une nouvelle politique de traitement des emprunts linguistiques (Office québécois de la langue française [OQLF], 2017), afin d'être en phase avec l'évolution sociolinguistique québécoise. Quelques mois plus tard, des articles apparaissaient dans la presse exprimant, sur le sujet, des opinions assez polarisées, comme c'est souvent le cas dans ce contexte où la question linguistique est étroitement liée à l'évolution et à l'identité de la société québécoise (Bouchard, 2002 : 14). Ainsi, pendant que les uns reprochaient à l'Office de prendre des décisions qui affaiblissent la langue française sans consulter la population (Dutrisac, 2017 ; Maurais, 2017a, 2017b) ou le critiquaient pour son désengagement de sa mission de normalisation et d'orientation de la langue au profit d'une action de description de l'usage (Corbeil et de Villers, 2017 ; Dutrisac, 2017 ; Maurais, 2017a ; Vincent, 2017), les autres rappelaient que les politiques ou guides de travail de l'organisme, quoique disponibles en ligne, se passent généralement du référendum citoyen ${ }^{1}$. Ils revendiquaient aussi le droit, voire le devoir, qu'a l'Office de s'adapter à l'évolution de la langue au Québec (Baillargeon, 2017 ; Cormier, 2017). Certains allaient jusqu'à voir dans ces critiques un possible règlement de comptes entre d'anciens artisans de l'Office, « groupe minoritaire de dissidents accrochés aux idées des années 70 » (Bergeron, 2017 : 29), et le personnel actuel de l'organisme ${ }^{2}$. Quoi qu'il en soit et bien que cette polémique n'ait pas semblé intéresser le grand public, elle a contribué à la tenue d'un colloque consacré à la perception des anglicismes au Québec ("A-t-on encore peur des anglicismes? », Sherbrooke, les 24 et 25 mai 2018), d’où est issue la présente étude.

Grâce aux recherches sur l'implantation terminologique, nous avons conscience que la reconnaissance et l'intégration de l'usage sont, depuis longtemps, étroitement associées au rôle d'orienteur de la langue que tient l'Office. En effet, elles découlent de l'approche sociale de l'aménagement linguistique et terminologique québécois que l'Office a adoptée dans les années 1980, dans l'espoir de voir les termes qu'il propose s'implanter dans les pratiques langagières des usagers. Guidé par cet objectif ultime de l'implantation des termes, l'Office a procédé à la révision de plusieurs de ses politiques et de ses guides de travail et s'est engagé dans diverses initiatives qui ont mis l'accent sur un rapprochement entre ses décisions terminologiques, l'usage et les usagers. C'est le cas du projet «En bons termes » que nous présentons ici. Mené dans le cadre de notre recherche de doctorat ${ }^{3}$, ce projet a fait collaborer en ligne des locuteurs intéressés à participer à l'enrichissement de la langue française avec les grands acteurs de l'aménagement terminologique au Canada réunis au sein du Comité de terminologie de Radio-Canada (désormais, CTRC), et dont l'Office est membre. Pendant

1. En effet, si les politiques et guides de travail « sont mis à la disposition des usagers », ils ne le sont qu'à titre informatif pour les « renseign[er] sur la manière dont se conçoit et se pratique la terminologie à l'Office » (OQLF, 2012b).

2. Voir l'article de G. Bernard Barbeau et V. Durocher dans le présent numéro.

3. Nous remercions les organismes et institutions suivants pour leur soutien financier de notre programme d'études: Conseil de recherches en sciences humaines du Canada ; Gouvernement de l'Ontario ; Université d'Ottawa; Ministère de l'Éducation de la Colombie-Britannique. 
quatre mois, les internautes ont proposé leurs solutions à divers problèmes terminologiques posés par le CTRC, qui les a considérées et évaluées pour parvenir à ses décisions et à la rédaction de fiches terminologiques.

Par sa participation au projet, l'Office confirme sa volonté d'inclure les utilisateurs des termes qu'il diffuse au sein du processus d'aménagement de la langue, approche qui s'inscrit dans une attitude plus générale d'ouverture sur l'usage à des fins d'implantation réussie des termes. Dès lors, il nous paraît intéressant de vérifier le traitement accordé par l'organisme aux termes suggérés par les internautes. Après un retour sur l'origine de l'approche sociale de l'aménagement terminologique de l'Office, sur sa justification pour garantir l'implantation des termes et sur l'impact qu'elle a eu sur diverses politiques et guides de travail, nous montrerons comment le projet «En bons termes » vise la collaboration avec les locuteurs pour mieux orienter l'usage et nous tenterons de répondre à la question suivante: dans quelle mesure les termes suggérés par les internautes ont-ils intégré les fiches terminologiques de l'Office produites à la suite du projet « En bons termes » et quel statut d'acceptabilité leur a-t-on donné ? Poury répondre, nous nous concentrerons sur les fiches publiées dans le GDT à l'issue du projet et qui, par conséquent, sont passées sous la loupe décisionnelle de l'Office. Nous souhaitons que les résultats de cette modeste étude apportent quelques indices sur la manière dont l'Office s'ouvre aux propositions d'usagers et dont il exerce son rôle d'orienteur de l'usage.

\section{Contexte}

\subsection{L'implantation, objectif ultime de l'aménagement terminologique québécois}

Au début des années 1960, alors que l'État québécois s'engage à préserver et à développer le français dans le milieu du travail et dans l'espace public de la province, l'aménagement terminologique apparaît rapidement comme une évidence, puisque l'anglais devait sa prédominance à « l'emploi et la diffusion de la langue et de la terminologie anglaises, totalement au niveau de la direction des entreprises, largement au niveau de l'exécution ou de la commercialisation » (Corbeil, 2007 : 94). La charge de fournir la terminologie française équivalente est alors confiée à l'Office, dès sa création en 1961. Depuis, l'organisme a accompli de nombreux travaux d'envergure dans ce domaine qui jouissent d'une assez heureuse renommée, comme peuvent le prouver l'intégration de plus en plus fréquente des trouvailles terminologiques québécoises dans les bases de données francophones ou dans les grands ouvrages lexicographiques que sont le Robert ou le Larousse.

Toutefois, en contexte d'aménagement terminologique, où l'orientation d'un certain usage est visée, il importe surtout de s'assurer que les termes et expressions proposés sont bien reçus, employés et adoptés par les usagers auxquels ils se destinent. Or, dès les années 1980, les premières enquêtes d'implantation terminologique qui avaient pour but de vérifier l'ampleur de l'usage des terminologies de l'Office révélaient que ces dernières étaient largement soit méconnues des locuteurs soit 
rejetées par eux à la faveur de l'anglicisme ou d'une forme considérée fautive (pour une recension de ces études, voir les travaux de Quirion, 2000, 2003 ou 2013). Ainsi, bien que «l'obligation [ait été] faite à l'Administration par la Charte de la langue française d'utiliser les termes et expressions normalisés par l'Office » (René, 2001 : 6), leur taux de succès s'élevait, selon les chercheurs, à quelque 27 \% (René, 2001 : 7), ce qui révélait que l'officialisation des termes ne suffisait pas à en faciliter l'implantation. Pour expliquer en partie cet échec, les enquêtes pointaient la longueur, l'aspect compliqué et la connotation péjorative des termes (Heller et al., 1982) ou le fait que les terminologies, qui se basaient sur une norme franco-européenne dans laquelle les locuteurs ne se reconnaissaient pas, soient méconnues ou inadaptées au contexte sociolinguistique québécois (Aléong et al., 1981; Chrétien, 1981).

Ces enquêtes ont permis de se questionner sur la nécessité de «"terminologiser" si l'on est peu soucieux d'implanter et d'implanter efficacement » (Chrétien, 1981 : 159) et ont poussé l'Office à investir dans de nouvelles stratégies d'implantation (René, 2001 : 7), notamment à adopter une approche socioterminologique ${ }^{4}$ qui se mette au diapason de la société qu'il sert et à mieux diffuser les terminologies qu'il officialise.

\subsection{Les politiques et guides de travail de l'Office}

Les politiques et guides de travail que l'Office a adoptés par la suite, telles celle relative aux québécismes (1985) ou celle à l'égard de l'emprunt linguistique (1980, 2007, 2017), ont reflété sa volonté d'« adapter [ses] orientations et [ses] méthodes de traitement en considérant l'évolution de la situation sociolinguistique au Québec» (OQLF, 2007: 3), à des fins d'implantation réussie des termes. C'est ce qu'il confirmait, en effet, lors de la présentation de sa nouvelle politique de l'emprunt linguistique au Congrès de l'OPALE ${ }^{5}$ :

[L]a société change et force est de constater que les locuteurs et les locutrices n'ont plus aujourd'hui le même rapport à la langue - aux langues - qu'il y a 50 ans, voire 15 ans. Les décisions qui ne tiennent pas compte de l'usage réel et valorisé, et le contredisent parfois, ne servent pas toujours l'atteinte des objectifs en aménagement linguistique. (...) L’aménagement ne peut plus se faire en vase clos, à la recherche d’un idéal langagier. (...) En fait, pour que l'Office mène à bien sa mission de francisation, il est nécessaire que son message soit

\footnotetext{
4. Apparue vers la fin des années 1980 et développée dans les années 1990, la socioterminologie est un courant théorique qui prône le besoin d'offrir une terminologie conforme aux valeurs sociolinguistiques des locuteurs à qui elle se destine; l'acceptation de terminologies en concurrence pour un usage social et fonctionnel; bref, la nécessité de voir le terme « dans son fonctionnement, sur le terrain des contradictions sociales » et non seulement « par rapport à un système» (Gambier, $1987: 320)$.

5. L'OPALE est le réseau des Organismes francophones de politique et d'aménagement linguistique. Il réunit les organismes des régions suivantes: la Belgique (Conseil de la langue française et de la politique linguistique de la Fédération Wallonie-Bruxelles), la France (Délégation générale à la langue française et aux langues de France), la Suisse (Délégation suisse à la langue française) et le Québec (Office québécois de la langue française), ainsi que l'Organisation internationale de la Francophonie en tant qu'observatrice. Les membres de l'OPALE se réunissent annuellement pour discuter des divers enjeux entourant l'aménagement de la langue française.
} 
non seulement entendu, mais également reçu et, surtout, relayé par les locuteurs. Dans un tel contexte, puisque les travaux d'aménagement linguistique effectués à l'Office ont pour objectif ultime de voir s'implanter les termes qu'il propose, la stratégie terminolinguistique la plus sensée en matière de traitement de l'emprunt est certainement de s'adapter à la réalité sociolinguistique actuelle et d'effectuer les changements qui s'imposent, sans trahir, évidemment, l'esprit du mandat qui lui est confié par la loi. (Maltais et Pedneault, 2016 : 278-279)

Parallèlement à la prise en compte du contexte sociolinguistique dans et pour lequel il travaille, l'Office a porté une attention toute particulière à la diffusion rapide de ses termes (René, 2001) et a, à cet égard, adopté une politique concernant l'officialisation linguistique et terminologique (OQLF, 2004). Celle-ci limite la normalisation et la recommandation pour privilégier la proposition, forme plus souple d'intervention, qui « consiste principalement à suggérer rapidement [aux] usagers des termes et expressions répondant à de nouveaux besoins de communication » (OQLF, 2004 : 6). Pour soutenir ce besoin de diffusion, l'Office a misé sur le GDT, le rendant accessible gratuitement en ligne depuis 2000 et en faisant un outil flexible, qui reflète l'évolution de l'usage (OQLF, 2004 : 13) puisque les fiches qui y sont déposées peuvent être - et sont - modifiées, aussi fréquemment que nécessaire.

\subsection{Le Comité de terminologie de Radio-Canada (CTRC)}

L'approche basée sur la « diffusion juste-à-temps »(OQLF, 2004 : 12) s'est couplée d'une volonté d'élargir le champ de dissémination des termes et amène l'Office à collaborer plus étroitement avec les médias, d'où sa participation au CTRC. Créé en 1998, puis relancé en 2012 après quelques années d'inactivité, le CTRC est composé de terminologues et de linguistes de la Société Radio-Canada, de l'Office, du Bureau de la traduction, mais aussi, plus récemment, de la direction éditoriale du dictionnaire Usito ${ }^{6}$ et de professeurs de l'Université d'Ottawa. Ensemble, ces experts de la langue, que nous dénommerons désormais « aménageurs » ${ }^{7}$, relèvent les concepts émergents dans la presse ou ailleurs et proposent des solutions de dénomination en français, qu'ils relayent ensuite sur leurs plateformes et dans leurs réseaux respectifs (Lafrance et Gourd, 2004). Leur travail porte sur ce que Quirion (2012) appelle la « néologie aménagiste », qui sert « soit [à] nommer un nouveau concept (...) soit [à] remplacer un vocable considéré inadéquat » (Quirion, 2012 : 131) dans un contexte d'orientation de l'usage. Pour résumer l'intérêt de sa participation au CTRC, l'Office explique que « [l]es travaux de ce comité permettent d'intervenir précocement et harmonieusement sur l'usage parce que les nombreux médias associés à l'enseigne de Radio-Canada, qui est partie prenante des choix terminologiques, en assurent une large diffusion auprès des locuteurs » (Turcotte, 2013 : 5).

\footnotetext{
6. Dictionnaire en ligne développé à l'Université de Sherbrooke et dédié à « décrire le français standard en usage au Québec, tout en faisant le pont avec le reste de la francophonie » (Usito, 2017).

7. Nous reprenons ici le terme retenu par Taifi (2012), suite à son analyse du concept d'aménagement en contexte linguistique.
} 


\subsection{Collaborer pour mieux implanter}

Les stratégies de diffusion énoncées précédemment ont leur utilité pour accroître la visibilité des propositions et mettre les usagers au courant de leur existence. En revanche, pour qu'un terme s'implante, il faut que les usagers en aient plus qu'une simple connaissance passive : il est nécessaire qu'ils l'adoptent et l'utilisent dans diverses situations de communication. Le succès de tout changement terminologique planifié dépend bel et bien de l'adhésion des utilisateurs des termes et expressions proposés (Quirion, 2014) et les acteurs de l'aménagement linguistique et terminologique auraient donc « avantage à cultiver un étroit contact avec les locuteurs » (Quirion, 2014 : 111). En effet, il semblerait qu'une collaboration avec les usagers, au cours de laquelle ceux-ci investissent de leur temps et de leur savoir pour l'élaboration des terminologies, les rende plus prompts à adopter les termes et puisse faire « gagner d'avance 50 \% de l'implantation des résultats du travail » (Diki-Kidiri, 2007 : 7). Si en contexte d'aménagement linguistique et terminologique, la proposition d'un tel gain ne peut guère se refuser, de telles collaborations demeurent en pratique assez rares ${ }^{8}$. Les terminologues ne travaillent certes pas en vase clos et il leur arrive fréquemment de consulter des experts de domaines pour valider leur compréhension ou la définition de tel ou tel concept (Bourigault et Slodzian, 1999). Toutefois, les personnes qu'ils consultent ne deviennent pas nécessairement des « partenaires avec qui [ils] collabore[nt] dans l'élaboration de la terminologie » (Diki-Kidiri, 2007 : 16).

Ce constat nous a poussée à proposer aux aménageurs du CTRC, qui par leur partenariat ont déjà compris l'utilité de travailler ensemble pour offrir des solutions linguistiques à implantabilité ${ }^{9}$ augmentée, d'ajouter un maillon manquant à leur chaîne collaborative : le locuteur, usager des terminologies que le CTRC propose. Outre l'intérêt évoqué ci-dessus d'une telle collaboration, nos discussions avec les aménageurs en amont du projet ont révélé qu'eux-mêmes voyaient divers avantages à impliquer les locuteurs dans le processus d'élaboration de la terminologie et de la néologie (p. ex. : accès à des idées qui apportent un nouvel éclairage sur le terme ou la notion; découverte de nouveaux experts de domaines ; possibilité de faire valider des propositions terminologiques en amont de leur diffusion). En revanche, les rares expériences de collaboration avec le grand public qu'ils avaient vécues ou dont ils avaient pris connaissance avaient aussi suffi à créer de nombreuses réticences, craintes ou préjugés, dont nous avons tenu compte dans l'élaboration du projet «En bons termes » (p. ex. : laborieux tri des nombreuses suggestions plus ou moins utilisables des locuteurs ; exigence de temps et de ressources pas toujours disponibles; difficile contrôle [des dérives] de la dis-

\footnotetext{
8. À notre connaissance, en aménagement linguistique ou terminologique francophone, seuls Dupuis et Martin (1985) ont rapporté les données d'une expérience de consultation d'usagers, non experts de domaines, pour féminiser les titres de professions. Hormis cet article, nous notons que les initiatives appelant les locuteurs à collaborer pour élaborer la terminologie sont rares, ponctuelles, lancées sous forme de concours à l'occasion d'un événement particulier (la Francofête, p. ex.) et, surtout, non étudiées par la recherche pour en évaluer la méthodologie, les résultats ou les impacts sur l'implantation des termes.
}

9. Nous reprenons ici un terme employé par Auger pour désigner les terminologies qui possèdent « un bon potentiel d'implantation » dans l'usage (Auger, 1994 : 47). 
cussion au sein des grands groupes de locuteurs; nécessaire justification des choix terminologiques finaux pouvant entraîner d'interminables débats).

\section{Description du projet « En bons termes»}

Le projet collaboratif «En bons termes » s'est déroulé en ligne sur une période de quatre mois, débutant par une phase pilote de 15 jours (du 2 au 17 octobre 2017), suivie de 81 jours d'expérience (entre le 30 octobre 2017 et le 4 février 2018). Toute personne de 14 ans et plus pouvait s'inscrire gratuitement sur la plateforme en ligne ${ }^{10}$, en choisissant un pseudonyme et un mot de passe, en fournissant une adresse courriel et quelques informations démographiques (p. ex. : sexe; âge ; pays d'origine et de résidence) et en signant le formulaire de consentement à la recherche. Au total, 63 personnes ont participé au projet, dont 44 femmes et 19 hommes. Notre recrutement s'est fait par «boule de neige » (invitation de personnes qui en invitent d'autres et ainsi de suite) et grâce au soutien du Portail linguistique ${ }^{11}$, qui a repéré l'expérience et a participé à la faire connaître à ses abonnés sur le réseau social Twitter. La majorité des participants, que nous dénommerons désormais « internautes », étaient originaires du Canada (74,6\%) et près de $89 \%$ d'entre eux y résidaient au moment de leur participation au projet (v. Tableau 1). Dans les deux cas et sans grande surprise, la représentation québécoise était de loin la plus élevée (60,3\% des internautes étaient originaires du Québec et 58,7\% y résidaient). Parmi les quelque $25 \%$ d'internautes originaires d'autres pays que le Canada, $19 \%$ venaient de la France, tandis que les $6 \%$ restant étaient natifs de l'Algérie, des ÉtatsUnis ou du Royaume-Uni. Seuls 11 \% des internautes résidaient en dehors du Canada au moment de l'expérience, dont $6 \%$ en France et le reste au Royaume-Uni.

Tableau 1. Répartition des internautes selon leurs pays d'origine et de résidence

\begin{tabular}{|c|c|c|c|}
\hline \multicolumn{2}{|c|}{ Pays (province) } & $\begin{array}{c}\text { Origine des } \\
\text { internautes }\end{array}$ & $\begin{array}{c}\text { Résidence des } \\
\text { internautes }\end{array}$ \\
\hline Canada & & 47 & 56 \\
\hline & Québec & 38 & 37 \\
\hline & Ontario & 6 & 8 \\
\hline & Colombie-Britannique & 0 & 1 \\
\hline & Nouveau-Brunswick & 2 & 2 \\
\hline & Alberta & 0 & 1 \\
\hline & Non précisé & 1 & 4 \\
\hline France & & 12 & 3 \\
\hline Autres & & 4 & --- \\
\hline
\end{tabular}

10. Pour bâtir cette plateforme, nous avons utilisé le logiciel de forum libre phpBB (https://www.phpbb.com/).

11. Le Portail linguistique du Canada est un service de ressources linguistiques géré par le Bureau de la traduction et accessible à l'adresse suivante : https://www.noslangues-ourlanguages.gc.ca/fr/index. 


\begin{tabular}{|c|c|c|c|}
\hline \multicolumn{2}{|c|}{ Pays (province) } & $\begin{array}{c}\text { Origine des } \\
\text { internautes }\end{array}$ & $\begin{array}{c}\text { Résidence des } \\
\text { internautes }\end{array}$ \\
\hline & États-Unis & 1 & --- \\
\hline & Grande-Bretagne & 2 & 3 \\
\hline
\end{tabular}

Les internautes se situaient à 82,5\% dans la tranche d'âge de la population active (entre 26 et 65 ans), avec $12,7 \%$ représentant les plus de 65 ans. Le projet a attiré moins de $5 \%$ de jeunes de moins de 25 ans (v. Figure 1).

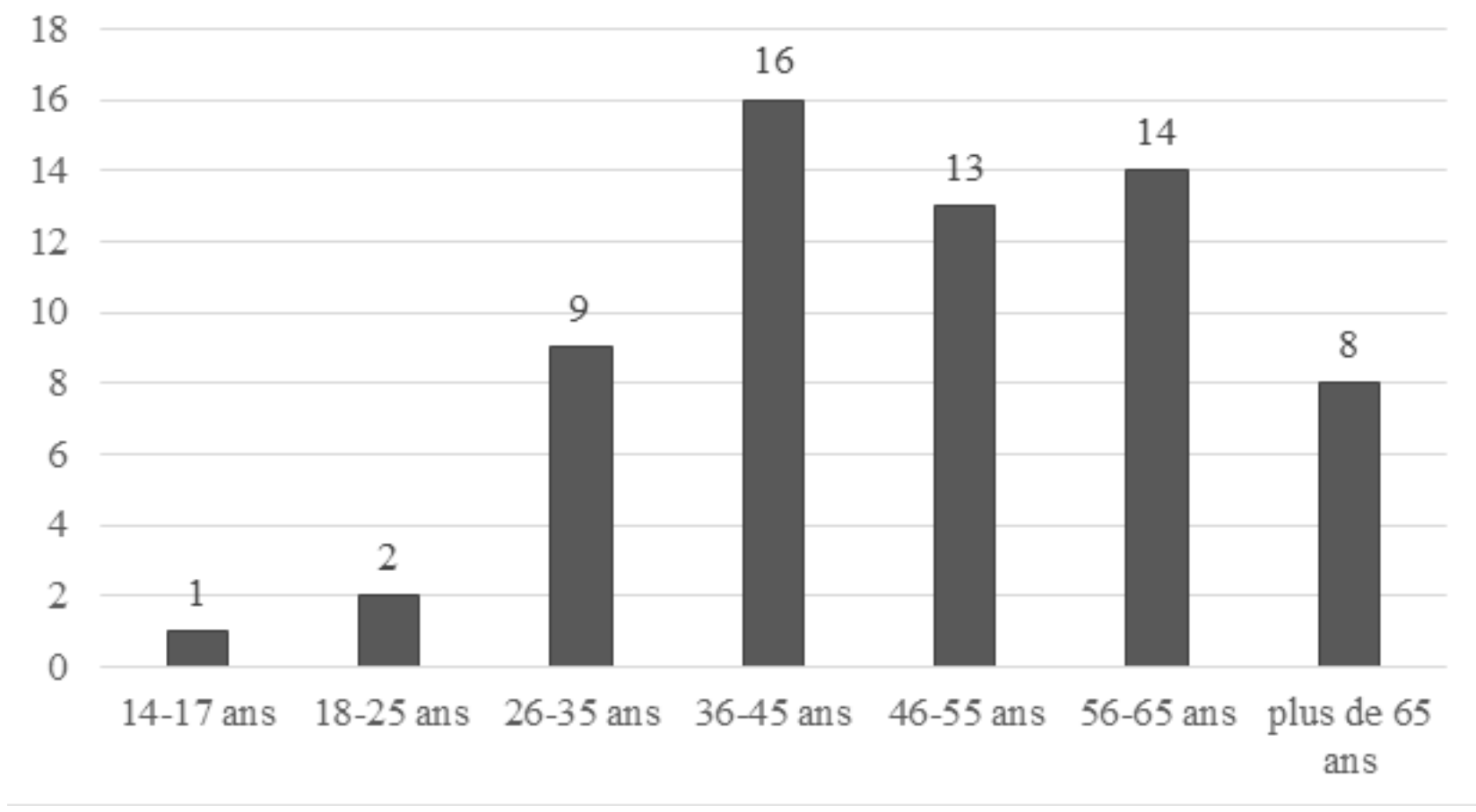

Figure 1. Répartition des internautes selon leur tranche d'âge

À quelques exceptions près, la collaboration entre les trois parties impliquées (les aménageurs du CTRC ; les internautes et nous, la chercheuse, qui avions endossé le rôle d'intermédiaire/modératrice) a suivi un cheminement identique pour traiter chaque concept (v. Figure 2). Tout d'abord, les aménageurs nous soumettaient le concept accompagné d'une définition, parfois d'exemples, de contextes et de suggestions préalables issues de leurs propres recherches (étape 1). Nous publiions ce problème sur le forum et nous invitions les internautes à offrir leurs suggestions pendant une durée déterminée d'entre 3 à 6 jours (étape 2). Une fois ce remue-méninges terminé (étape 3), nous compilions toutes les suggestions dans un sondage (étape 4) et donnions entre 24 et 48 heures aux internautes pour sélectionner les trois suggestions qui leur plaisaient le plus ${ }^{12}$ (étape 5). Nous

12. Cette approche donnait, d'une part, la possibilité aux internautes qui n'avaient pas fait de suggestions terminologiques à l'étape $\mathbf{2}$ de participer à l'expérience en faisant valoir leurs préférences, mais aussi de regrouper les voix de ceux qui s'étaient exprimés individuellement. 
utilisions alors les résultats de ce vote pour faire suivre toutes les suggestions des internautes aux aménageurs, classées dans l'ordre décroissant de leurs préférences ${ }^{13}$ (étape 6). Les aménageurs prenaient ensuite le temps nécessaire pour évaluer les suggestions des internautes et choisissaient ou non de s'en inspirer pour parvenir à leurs décisions finales. Au terme de leur réflexion, ils partageaient une courte rétroaction et une indication de leurs choix finaux (étape 7 ), que nous publiions sur le forum ${ }^{14}$ à l'attention des internautes (étape 8).

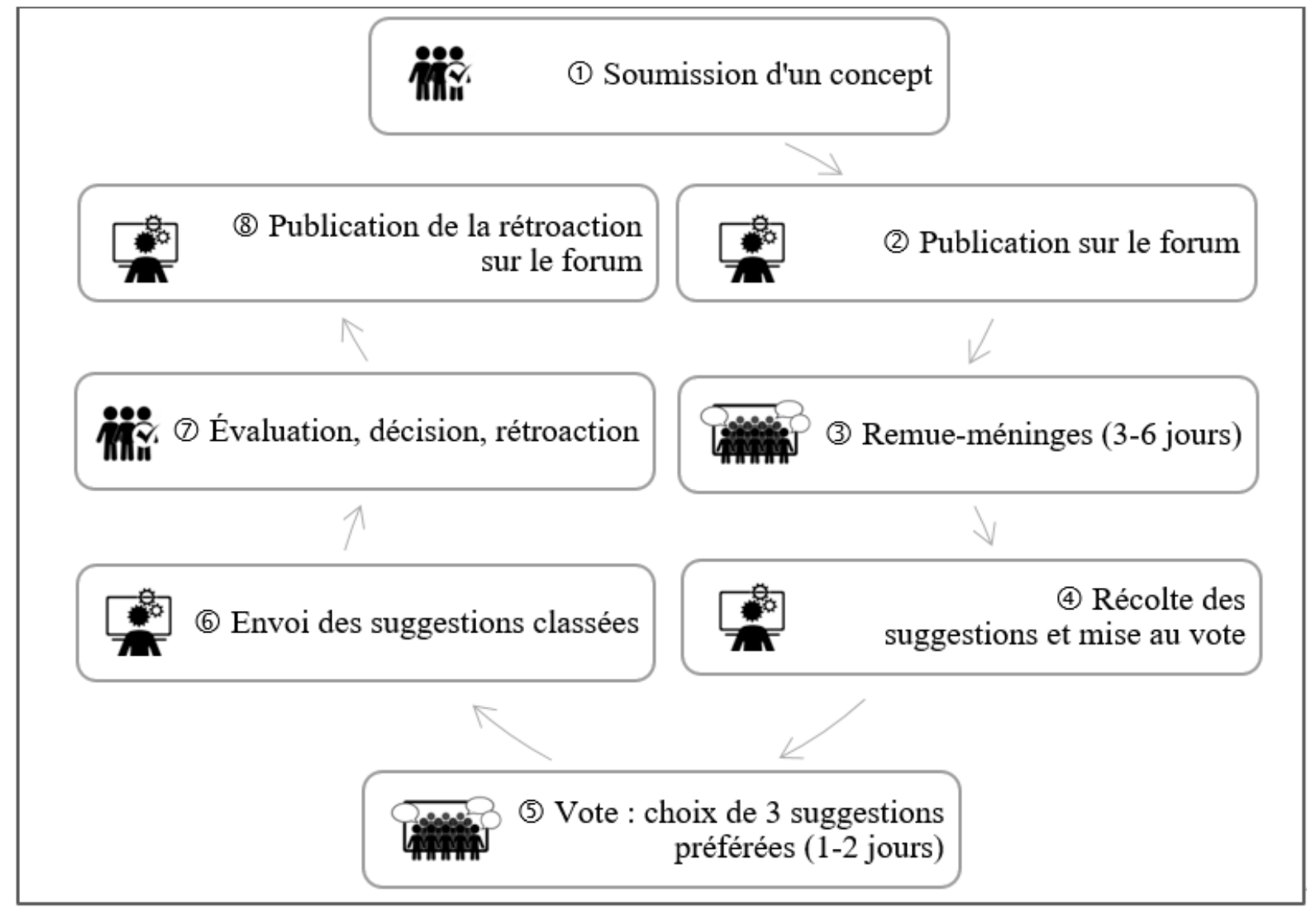

Figure 2. Déroulement de la collaboration au sein du projet « En bons termes »

Au total, 24 concepts ont été discutés sur le forum et un total de 17 fiches terminologiques ont été créées, ce qui montre qu'en somme, les aménageurs ont bien intégré le projet au sein de leur routine de travail. Selon les cas, ces fiches ont été publiées dans l'ensemble des banques de données terminologiques gérées par les aménageurs (dans le GDT pour l'Office, dans Termium Plus pour le Bureau de la traduction ou dans sa banque interne pour la Société Radio-Canada) ou dans certaines d'entre elles.

13. Dans deux cas (save-the-date card et water square), l'étape $\mathbf{5}$ du vote n'a pas eu lieu et les suggestions des internautes ont alors été classées dans un ordre alphabétique avant d'être envoyées aux aménageurs du CTRC.

14. Notons que les rétroactions étaient publiées sous forme de messages fermés à la discussion et donc, uniquement à titre informatif. 


\section{Méthodologie}

Nous limitons notre étude aux fiches publiées dans le GDT et qui, de ce fait, sont passées entre les mains des terminologues de l'Office et ont fait l'objet d'un traitement qui répond aux critères énoncés dans les politiques qui guident le travail de l'organisme. Notre analyse concerne donc 27 termes répartis sur 11 fiches terminologiques (v. Annexe), dont nous observerons à la fois l'origine et le statut d'acceptabilité. Les fiches du GDT étant régulièrement révisées par l'Office, nous précisons que les termes de notre étude sont issus des fiches telles qu'elles apparaissaient le 4 avril 2018, date à laquelle nous avons terminé la collecte de nos données.

\subsection{Origine des termes des fiches}

Nous avons défini trois catégories pour identifier l'origine des termes qui apparaissent dans les fiches du GDT. La première (catégorie A) renvoie aux créations terminologiques des aménageurs. On y trouve les termes ajoutés à l'étape 7 (v. Figure 2), au moment de la rédaction de la fiche, ainsi que ceux issus des recherches préliminaires et soumis aux internautes à l'ouverture de la discussion sur le forum. Ces derniers en prenaient connaissance et, lors de la discussion (étape 3 ), les validaient, les critiquaient ou les ignoraient pour offrir leurs propres suggestions. La seconde catégorie (catégorie B) décrit les suggestions des internautes qui ont été intégrées aux fiches sans aucune modification de la part des aménageurs. Finalement, la troisième catégorie (catégorie C) fait référence aux termes des fiches qui proviennent en partie des suggestions des internautes. Pour sélectionner ces termes, nous nous sommes appuyée sur les données et sur ce que nous avons pu observer pendant le déroulement du projet «En bons termes ». Les suggestions surlignées en gras dans l'Annexe sont celles qui, nous pensons, ont influencé les décisions finales des aménageurs. Nous justifions nos choix dans le Tableau 2, mais reconnaissons que ce classement comporte une part de subjectivité non négligeable. Par exemple, on pourra s'étonner que, pour le concept mudroom, «vestiaire d'entrée » ne se trouve pas dans la catégorie $C$, alors que plusieurs suggestions d'internautes contiennent le substantif « vestiaire ». Notre choix repose sur le fait que les aménageurs, souhaitant découvrir lequel de « vestiaire » ou de « vestibule » évoquait le mieux la notion mudroom pour les internautes, avaient inclus ces deux termes dans la définition. Par conséquent, alors que « vestibule de rangement »a été placé dans la catégorie B parce que les aménageurs l'ont repris dans son intégralité parmi les suggestions des internautes pour l'ajouter à la fiche, nous considérons que « vestiaire d'entrée » est un terme de catégorie A puisque les deux éléments qui le composent sont le produit du travail des aménageurs. Il en va de même pour le concept slasheur : le préfixe « pluri » et le radical «cumul » apparaissent dans certaines suggestions d'internautes (v. Annexe), mais nous avons jugé qu'elles restent trop éloignées des décisions des aménageurs pour être considérées de catégorie C. 
Tableau 2. Justification de la sélection des termes de catégorie C (à lire avec l'Annexe)

\begin{tabular}{|c|c|c|}
\hline Concept traité & $\begin{array}{l}\text { Terme(s) de catégorie } C \\
\text { inscrit(s) dans la fiche }\end{array}$ & Justification \\
\hline crowd gaming & jeu participatif de foule & $\begin{array}{l}\text { «jeu participactif de foule » et « jeu participatif » ont été } \\
\text { proposés par les internautes. }\end{array}$ \\
\hline save the date & $\begin{array}{l}\text { réservez cette date } \\
\text { date à retenir }\end{array}$ & $\begin{array}{l}\text { Les internautes ont proposé « réservez la date » qui a été } \\
\text { repris tel quel dans la fiche (catégorie B), ainsi qu'une } \\
\text { suggestion avec l'adjectif démonstratif « cette». De } \\
\text { même, « date à réserver » et « retenir ou retenez la date » } \\
\text { ont été proposés par les internautes. }\end{array}$ \\
\hline save-the-date card & carton de préinvitation & $\begin{array}{l}\text { Les internautes ont proposé « carte de préinvitation » } \\
\text { qui a été repris tel quel dans la fiche (catégorie B), ainsi } \\
\text { qu’une suggestion incluant le substantif « carton ». }\end{array}$ \\
\hline \multirow{3}{*}{ water square } & $\begin{array}{l}\text { place inondable } \\
\text { place publique } \\
\text { inondable }\end{array}$ & \multirow{3}{*}{$\begin{array}{c}\text { Les suggestions des internautes ont entraîné la créa- } \\
\text { tion de quatre fiches, l'une renvoyant au concept water } \\
\text { square et trois autres à des concepts connexes. Les } \\
\text { aménageurs ayant clairement indiqué avoir été inspirés } \\
\text { par les suggestions des internautes pour la création de } \\
\text { ces trois fiches imprévues, nous avons inclus tous les } \\
\text { termes qui s'y trouvent soit dans la catégorie B soit dans } \\
\text { la catégorie C, à l'exception de «place multifonctionnelle } \\
\text { inondable » qui nous a paru plus fortement influencé par } \\
\text { les aménageurs et que nous avons placé dans la catégo- } \\
\text { rie A. }\end{array}$} \\
\hline & $\begin{array}{l}\text { espace publique } \\
\text { inondable }\end{array}$ & \\
\hline & $\begin{array}{l}\text { jardin pluvial } \\
\text { jardin inondable }\end{array}$ & \\
\hline $\begin{array}{l}\text { zero-acreage } \\
\text { farming }\end{array}$ & $\begin{array}{l}\text { agriculture urbaine à surface } \\
\text { nulle }\end{array}$ & $\begin{array}{c}\text { «agriculture urbaine » a été proposé par les internautes, } \\
\text { qui ont aussi souligné l'idée «sans espace au sol » dans } \\
\text { certaines de leurs suggestions. }\end{array}$ \\
\hline
\end{tabular}

\subsection{Statut d'acceptabilité des termes dans les fiches}

L'Office, en raison de son rôle normatif et d'orientation de l'usage, a recours à un code couleur et à diverses notes pour indiquer le degré d'acceptabilité des termes et préciser leurs contextes d'emploi (OQLF, 2012a, 2015). Pour identifier le statut des termes de notre étude, nous avons repris la terminologie propre au GDT. Ainsi, le terme vedette, placé en entrée de fiche, correspond au terme dont l'Office recommande l'emploi dans tous les contextes de communication. Les termes privilégiés, codés en vert dans la fiche, sont eux aussi acceptés dans tous les contextes pour désigner la notion. Ils reprennent donc le terme vedette (que nous n'avons pas comptabilisé deux fois dans les données que nous présentons ci-dessous) et incluent, s'il y a lieu, d'autres formes ou termes interchangeables. Certains termes des fiches du GDT sont codés en jaune, pour indiquer qu'ils devraient être réservés à certains contextes de communication, ou en rouge, pour signaler qu'ils sont déconseillés. Ces termes sont généralement accompagnés d'une explication ou de marques d'usage (courant, familier, critiqué, etc.). Finalement, les fiches peuvent contenir des notes qui sont ajoutées sous la définition et apportent diverses informations aidant à mieux cerner la notion. 


\section{Présentation et discussion des résultats}

\subsection{Origine des termes présents dans les fiches du GDT}

Nous avons divisé les résultats en deux groupes, le premier représentant les cas où les concepts ont été soumis aux internautes accompagnés de suggestions préalables des aménageurs et le second renvoyant aux cas où les concepts ont été soumis sans suggestion (v. Tableau 3).

Tableau 3. Origine des termes présents dans les fiches du GDT selon les concepts traités

\begin{tabular}{|c|c|c|c|}
\hline \multirow{2}{*}{$\begin{array}{c}\text { Concepts avec suggestions } \\
\text { préalables }\end{array}$} & \multicolumn{3}{|c|}{ Origine des termes } \\
\hline & Catégorie $\mathbf{A}$ & Catégorie B & Catégorie $\mathrm{C}$ \\
\hline save the date & 1 & 2 & 2 \\
\hline save-the-date card & 1 & 1 & 1 \\
\hline tap takeover & 3 & --- & --- \\
\hline $\begin{array}{c}\text { zero acreage } \\
\text { farming/Z-farming }\end{array}$ & --- & --- & 1 \\
\hline sous-total & $\begin{array}{c}5 \\
(42 \%)\end{array}$ & $\begin{array}{c}3 \\
(25 \%)\end{array}$ & $\begin{array}{c}4 \\
(33 \%)\end{array}$ \\
\hline \multirow{2}{*}{$\begin{array}{l}\text { Concepts } \\
\text { sans suggestion } \\
\text { préalable }\end{array}$} & \multicolumn{3}{|c|}{ Origine des termes } \\
\hline & Catégorie A & Catégorie B & Catégorie C \\
\hline crowd gaming & 1 & 1 & 1 \\
\hline mudroom & 1 & 1 & --- \\
\hline slasheur & 2 & --- & -- \\
\hline water square & 1 & --- & 2 \\
\hline espace public inondable & -- & -- & 1 \\
\hline jardin pluvial & --- & 1 & 2 \\
\hline parcinondable & -- & 1 & --- \\
\hline Sous-Total & $\begin{array}{c}5 \\
(33 \%)\end{array}$ & $\begin{array}{c}4 \\
(27 \%)\end{array}$ & $\begin{array}{c}6 \\
(40 \%)\end{array}$ \\
\hline Total & $\begin{array}{c}10 \\
(37 \%)\end{array}$ & $\begin{array}{c}7 \\
(26 \%)\end{array}$ & $\begin{array}{c}10 \\
(37 \%)\end{array}$ \\
\hline
\end{tabular}

\subsubsection{Origine des termes du groupe 1 : avec suggestions préalables des aménageurs}

Pour les quatre concepts de ce groupe, les aménageurs ont soumis, lors de l'étape 1 du processus, un total de neuf suggestions terminologiques, dont cinq ont trouvé leur place dans les fiches du GDT (v. Annexe). Ainsi, save the date et save-the date card étaient accompagnées de deux et d'une suggestions préalables respectivement, dont deux ont été conservées (" prenez date » et " préinvi- 
tation »). Quatre suggestions étaient fournies au moment du lancement de la discussion au sujet du concept tap takeover, dont trois forment le contenu intégral de la fiche finale. Finalement, la notion zero-acreage farming ou z-farming avait été envoyée aux internautes avec deux suggestions, qui n'apparaissent ni l'une ni l'autre dans la fiche publiée. Au total, ces propositions de catégorie A, issues du travail préliminaire des aménageurs, représentent $42 \%$ des termes intégrés aux fiches de ce groupe. Il s'agit d'un taux de rétention assez élevé qui révèle, d'une part, que ces suggestions ne sont pas le résultat d'un simple remue-méninges, mais plutôt celui d'une recherche assez aboutie, et, d'autre part, qu'elles sont dès le départ envisagées par les aménageurs comme des solutions terminologiques valables et adéquates, voire finales.

En soumettant ces termes à l'étape $\mathbf{1}$, on peut s'attendre à ce que le CTRC veuille vérifier leur implantabilité et, au besoin, se donner la chance de les réviser avant leur diffusion et, donc, avant qu'ils n'atteignent un public plus large. Dans les trois cas où un vote a eu lieu à l'étape $\mathbf{5}$ (v. Annexe), on constate que ces suggestions ne sont pas véritablement approuvées par les internautes. Ainsi, dans le cas de zero-acreage farming/Z-farming, l'une des propositions, " agriculture sur le bâti urbain », n'a reçu aucun vote, tandis que l'autre, « agriculture en bâti urbain », a récolté moitié moins de votes (15\%) que la suggestion arrivée en première place et ayant inspiré la vedette de la fiche. Le faible taux de soutien à leurs propositions lors du vote aura donc visiblement incité les aménageurs à mettre leurs idées initiales de côté pour créer une fiche tenant compte des préférences des internautes. Toutefois, cette approche ne vaut ni pour save the date alors que la vedette de la fiche (" prenez date ») ne reçoit que $6 \%$ des votes contre $17 \%$ pour l'expression «à vos agendas », ni pour tap takeover, dont les trois termes retenus dans la fiche se placent en seconde position avec cinq autres suggestions. On peut donc conclure que les questions d'implantabilité et de prise en compte de l'opinion des usagers que sont ici les internautes ne suffisent pas à justifier le rejet de termes que les aménageurs jugent adéquats.

Cela ne signifie pas pour autant qu'aucune considération pour les suggestions d'internautes n'ait été donnée de la part des aménageurs. On remarque, en effet, que 25 \% des 12 termes qui apparaissent dans les fiches de ce premier groupe sont le produit intégral de la réflexion et de la créativité des internautes (termes de la catégorie B) et 33 \% en sont le produit partiel (termes de la catégorie C). Ainsi, les propositions « carte de préinvitation » pour save-the-date card, ainsi que « réservez la date » et « à vos agendas » pour save the date sont directement issues des suggestions d'internautes. Pour ces mêmes fiches, les aménageurs ont aussi adapté trois suggestions d'internautes pour proposer « carton de préinvitation », d'une part, et « réservez cette date » et « date à retenir », d'autre part. Finalement, rappelons que le terme vedette de la fiche pour zero-acreage farming/Z-farming est aussi un terme de la catégorie C. Il nous semble donc évident que les aménageurs, en général, et l'Office, en particulier, puisqu'il s'agit ici de fiches qu'il a entérinées, ont pris le temps de consulter et d'évaluer les suggestions des internautes. Ce faisant, ils leur ont réservé un bon accueil au sein de leurs décisions finales, même si le CTRC disposait déjà de propositions acceptables à l'issue de ses propres recherches préliminaires. 


\subsubsection{Origine des termes du groupe 2 : sans suggestion des aménageurs}

Sur les 15 termes répartis dans les fiches de ce second groupe (v. Tableau 3), cinq sont de catégorie A et dix de catégorie B ou C, ce qui démontre de nouveau une réelle prise en compte des suggestions des internautes de la part des aménageurs. D’ailleurs, trois fiches ne contiennent aucun terme de catégorie A. Il est, cependant, utile de préciser qu'il s'agit des fiches que les aménageurs ont créées après avoir constaté que plusieurs termes suggérés par les internautes pour water square prêtaient à confusion, renvoyaient à des concepts connexes et méritaient donc d'avoir leur propre fiche terminologique. Si nous mettons de côté ces fiches imprévues qui, comme nous l'avons expliqué dans le Tableau 2, font la part belle aux suggestions des internautes, nous remarquons que pour les concepts crowd gaming, mudroom, slasheur et water square, au moins un terme de catégorie A a été ajouté à chacune des fiches finales et que celle du concept slasheur ne comporte aucune proposition provenant soit entièrement soit partiellement des internautes. Les trois autres fiches contiennent, pour leur part, un total de deux termes de catégorie B et de trois termes de catégorie C, égal à celui des termes de catégorie A. Les aménageurs ont, comme nous l'avons observé précédemment avec les fiches du groupe 1, pris en considération les suggestions des internautes et leur ont donné une place identique dans les fiches finales à celle occupée par leurs propres propositions. Par contre, à l'exception des fiches imprévues mentionnées ci-dessus, les termes des catégories B et $C$ ne forment jamais à eux seuls les fiches finales et sont toujours accompagnés de propositions de catégorie A, ce qui suggèrerait que les fiches ne peuvent se passer des propositions des spécialistes de la terminologie et de la néologie pour être considérées valides. Une analyse des critères formels et sémantiques des suggestions des internautes serait évidemment nécessaire pour vérifier le bien-fondé de cette supposition énoncée sur la base de données chiffrées, car, comme le montre le concept tap takeover, les suggestions préférées des internautes ne garantissent pas qu'elles soient correctes d'un point de vue terminologique (v. Annexe).

\subsection{Statut d'acceptabilité des termes dans les fiches du GDT}

L'approche normative adoptée par l'Office donne aux termes présents dans les fiches qu'il publie des indications sur leur acceptabilité (v. section 4.2). Ainsi, puisque la présence d'un terme sur une fiche ne signifie pas nécessairement qu'il s'agisse d'un terme dont l'Office recommande l'usage, nous avons jugé intéressant de vérifier le statut que les aménageurs ont donné aux termes issus du projet «En bons termes » dans les fiches du GDT, selon leur origine (v. Tableau 4). 
Tableau 4. Statut d'acceptabilité des termes présents dans les fiches du GDT selon leur origine

\begin{tabular}{|c|c|c|c|}
\hline \multirow{2}{*}{ Statut des termes } & \multicolumn{3}{|c|}{ Origine des termes } \\
\hline & Catégorie A & Catégorie B & Catégorie C \\
\hline $\begin{array}{c}\text { termes privilégiés (dont } \\
\text { les termes en vedette } \\
[\mathrm{v}])\end{array}$ & $\begin{array}{l}\text { - conquête des fûts } \\
\text { - cumulotravailleur(-se) } \\
\text { - incursion brassicole } \\
\text { - invasion brassicole (v) } \\
\text { - jeu coopératif de foule } \\
\text { - place multifonctionnelle } \\
\text { inondable } \\
\text { - préinvitation } \\
\text { - prenez date (v) } \\
\text { - travailleur(-se) } \\
\text { plurifonction (v) } \\
\text { - vestiaire d'entrée (v) }\end{array}$ & $\begin{array}{l}\text { - carte de préinvitation (v) } \\
\text { - jardin de pluie } \\
\text { - jeu interactif de foule (v) } \\
\text { - parc inondable (v) } \\
\text { - réservez la date }\end{array}$ & $\begin{array}{l}\text { - agriculture urbaine à } \\
\text { surface nulle }(v) \\
\text { - carton de } \\
\text { préinvitation } \\
\text { - espace public } \\
\text { inondable (v) } \\
\text { - jardin inondable } \\
\text { - jardin pluvial (v) } \\
\text { - jeu participatif de foule } \\
\text { - place inondable (v) } \\
\text { - place publique } \\
\text { inondable } \\
\text { - réservez cette date }\end{array}$ \\
\hline total & $\begin{array}{c}10 \\
\text { (4 en vedette) }\end{array}$ & $\begin{array}{c}5 \\
\text { (3 en vedette) } \\
\end{array}$ & $\begin{array}{c}9 \\
\text { (4 en vedette) }\end{array}$ \\
\hline $\begin{array}{l}\text { termes à utiliser dans } \\
\text { certains contextes }\end{array}$ & --- & $\begin{array}{l}\text { - à vos agendas } \\
\text { - vestibule de } \\
\text { rangement }\end{array}$ & - date à retenir \\
\hline total & 0 & 2 & 1 \\
\hline
\end{tabular}

Tous les termes consignés dans les fiches de notre étude portent soit la marque «privilégiés », dont le terme en vedette (v) fait partie, soit la marque « utilisés dans certains contextes ». Dans ce dernier groupe de termes, seul «vestibule de rangement » trouve sa place dans la zone en jaune de la fiche (v. Figure 3), alors que les deux autres termes sont mentionnés au sein d'une note qui se lit ainsi : «On peut également avoir recours à des expressions telles que date à retenir ou à vos agendas »(OQLF, 2018i). 


\begin{tabular}{|c|c|c|}
\hline vestiai & d'entrée & Anglais [EN] \\
\hline Domaines & $\begin{array}{l}\text { industrie de la construction }>\text { architecture } \\
\text { mobilier }>\text { meuble de rangement }\end{array}$ & \\
\hline Auteur & Office québécois de la langue française, 2017 & \\
\hline
\end{tabular}

\section{Définition}

Pièce ou espace généralement aménagé à l'entrée de la maison, servant de rangement pour les vêtements d'extérieur et autres objets.

\section{Note}

Le vestiaire d'entrée peut être une pièce indépendante attenante au vestibule ou un espace aménagé à l'intérieur de ce dernier. On peut y trouver des paniers de rangement, des crochets, un coffre, un casier, un banc. Dans certains cas, lorsque lespace le permet, le vestiaire d'entrée comporte une salle d'eau et une buanderie.

\section{Terme privilégié}

vestiaire d'entrée n.m.

Terme utilisé dans certains contextes

vestibule de rangement $\mathrm{n.m}$.

Le terme vestibule de rangement désigne plus précisément une pièce de transition entre l'extérieur et l'intérieur, aménagée en vestiaire.

Anglais

Auteur Office québécois de la langue française, 2017

Termes

mudroom

mud room

Figure 3. Capture d'écran de la fiche « vestiaire d'entrée » du GDT (datée du 4 avril 2018)

On ne s'étonnera pas de voir les 10 termes de catégorie A inclus dans la section des termes privilégiés. En effet, tout d'abord proposés par les aménageurs du CTRC, puis avalisés par l'Office, il y a de fortes chances pour qu'ils intègrent le GDT en tant que termes dont on préconise l'usage dans toutes les situations de communication. Cependant, le fait qu'aucun terme de catégorie A ne se trouve en zone jaune ou rouge indique aussi que les aménageurs n'ont pas identifié de termes à limiter à certains contextes ou inadéquats autres que ceux que les internautes ont pu leur fournir.

Les 17 suggestions d'internautes prennent, elles, des positions un peu plus variées au sein des fiches. Si trois suggestions (deux de catégorie B et une de catégorie C) ont été intégrées aux fiches en tant que termes à employer dans certains contextes uniquement, il faut noter que 14 d'entre elles, soit $82 \%$, jouissent d'un statut de termes privilégiés. On remarque, par contre, qu'elles sont en grande partie de catégorie C (9 suggestions sur 14) et ont donc surtout joué un rôle d'inspiration 
pour les aménageurs, qui les ont modifiées et ajustées afin de générer des termes adéquats. Cela expliquerait d'ailleurs pourquoi on trouve autant de termes placés en vedette dans la catégorie C que dans la catégorie A. Les suggestions des internautes de catégorie B sont, pour leur part, moins nombreuses à accéder au statut de termes privilégiés et, parmi elles, seules deux sont placées en vedette de fiches, laissant les trois autres jouer le rôle d'équivalents de la langue standard jugés adéquats par l'Office pour dénommer la notion (OQLF, 2012a). Au total, toutefois, 54 \% des fiches (6 sur 11) sont présentées par un terme vedette provenant de suggestions intégrales ou partielles des internautes, ce qui révèle à la fois que les aménageurs ont été ouverts aux suggestions des usagers présents sur « En bons termes », mais aussi que ces suggestions étaient visiblement acceptables et une bonne source d'inspiration pour leur travail.

\section{Conclusion}

Notre étude s'inscrit dans la lignée des recherches sur l'implantation des termes en aménagement linguistique et terminologique québécois, contexte où l'Office guide l'usage terminologique et évalue le succès de son action en mesurant le degré d'implantation des propositions qu'il diffuse. Pour parvenir à son objectif, l'organisme a adopté, au fil des ans, une approche socioterminologique qui tient compte de l'usage réel et valorisé au Québec (Maltais et Pedneault, 2016), ainsi que des valeurs sociolinguistiques de la province. Cela l'a amené à réviser plusieurs de ses politiques et guides de travail, dont fait partie la récente politique de l'emprunt linguistique (OQLF, 2017) qui a fait polémique à l'automne 2017 (Dutrisac, 2017 ; Corbeil et de Villers, 2017 ; Maurais, 2017a, 2017b ; Vincent, 2017) et qui a inspiré le thème du colloque où nous avons présenté notre travail. Il a aussi misé sur la diffusion plus rapide et plus large de ses propositions, ce que reflète son partenariat avec d'autres acteurs de l'aménagement linguistique canadien au sein du CTRC (Turcotte, 2013). Plus récemment, et alors que la recherche souligne que l'intégration des usagers visés dans le processus de création terminologique (Diki-Kidiri, 2007 ; Quirion, 2011) pourrait contribuer à l'implantation rapide et réussie des termes, l'Office a participé au projet «En bons termes ». Ce projet mené dans le cadre de notre doctorat a fait collaborer en ligne les aménageurs du CTRC avec un groupe de 63 internautes, entre octobre 2017 et février 2018. Au terme de cette expérience, 11 nouvelles fiches terminologiques ont été ajoutées au GDT et il nous a paru intéressant de vérifier, d'une part, la provenance des termes qu'elles contiennent, selon qu'il s'agit de propositions d'aménageurs ou d'internautes, et d'autre part, leur statut d'acceptabilité (termes privilégiés, à utiliser dans certains contextes ou déconseillés).

Nous avons pu constater que les fiches présentaient un nombre assez important (71\%) de termes de catégorie B et C, c'est-à-dire suggérés par les internautes (7 termes sur 27) et inspirés de suggestions d'internautes (10 termes sur 27), ce qui indique soit une réelle volonté d'inclure la voix des usagers dans le processus d'aménagement terminologique soit, tout simplement, que les suggestions des internautes étaient entièrement ou partiellement acceptables. De plus, un nombre identique de termes d'aménageurs (catégorie A) et de termes de catégorie C ont rejoint les fiches en tant que vedette, terme au statut d'acceptabilité le plus élevé. Bien que le classement des termes en catégo- 
rie $C$ ne soit pas dénué d'une certaine subjectivité (v. section 4.1), il nous semble qu'un tel résultat montre combien l'exercice collaboratif aura profité aux aménageurs, en leur offrant des pistes utiles pour l'élaboration de la terminologie. Cela se confirme particulièrement avec le cas des trois fiches imprévues renvoyant à des concepts connexes à water square. En effet, si d'un côté, la discussion autour de ce concept révèle que les termes proposés par les internautes ne renvoient pas à la bonne notion, de l'autre, cette confusion a mené à l'identification de notions importantes que les aménageurs auraient probablement manqué de repérer par eux-mêmes. Finalement, les aménageurs ont pu profiter de cette collaboration pour prendre le pouls d'un groupe d'usagers au sujet de termes qu'ils souhaitaient proposer pour dénommer certains concepts (v. groupe 1, Tableau 3). Si ces termes se retrouvent dans trois fiches sur quatre, des suggestions d'internautes viennent généralement aussi s'ajouter, voire, dans le cas de save-the-date card, prendre la vedette de la fiche. La collaboration avec les internautes semble donc avoir eu pour effet d'enrichir les fiches terminologiques publiées dans le GDT, même si la touche des aménageurs reste très présente soit par l'ajout de leurs propositions personnelles soit par l'adaptation des suggestions d'internautes.

En conclusion, une analyse quantitative de nos données indique une large intégration des suggestions des internautes dans les fiches terminologiques et, de ce fait, une appréciation réelle de leurs contributions pour l'enrichissement de la langue. Ainsi, tout en demeurant l'orienteur d'une norme que lui-même définit, l'Office fait preuve d'une approche d'aménagement terminologique qui se veut souple et accueillante des propositions d'usagers. Conscient que, "quand [les] propositions sont trop tranchées, il arrive que les gens trouvent que [l'Office n'a] pas de bon sens » (Turcotte, citée dans Baillargeon, 2017), l'organisme contribue par cette ouverture à une série d'efforts déployés depuis plusieurs années pour répondre à son mandat, tout en atteignant son objectif ultime : l'implantation réussie de ses termes et expressions. II serait, néanmoins, intéressant de comparer nos résultats aux opinions des participants à ce projet collaboratif pour répondre, notamment, aux questions suivantes : comment les internautes ont-ils perçu la manière dont les aménageurs ont traité leurs suggestions? Dans quelle mesure trouvent-ils que le contenu des fiches publiées dans le GDT répond à leurs besoins et attentes terminologiques? Quelle utilité ont réellement eue les suggestions des internautes pour les aménageurs ? Dans quelle mesure les aménageurs sont-ils disposés à instaurer une collaboration durable avec le grand public? Les réponses à ces questions permettraient certainement de nuancer l'étendue de l'ouverture ressentie dans notre modeste étude. Finalement, il sera nécessaire dans quelques années de faire valider l'hypothèse d'une meilleure implantation grâce à la collaboration avec les usagers (Diki-Kidiri, 2007 ; Quirion, 2011), en relevant combien de termes privilégiés issus des 11 fiches de notre étude se seront implantés dans l'usage. 


\section{Références}

\section{Fiches terminologiques du GDT}

Office québécois de la langue française [OQLF] (2018a), agriculture urbaine à surface nulle, disponible sur www.granddictionnaire.com/ficheOqlf.aspx?Id_Fiche=26543957. [Page consultée le 23 février 2019.]

OQLF (2018b), carte de préinvitation, disponible sur www.granddictionnaire.com/ficheOqlf.aspx?ld_Fiche=26543985. [Page consultée le 23 février 2019.]

OQLF (2018c), espace public inondable, disponible sur www.granddictionnaire.com/ficheOqlf. aspx?Id_Fiche=26544035. [Page consultée le 23 février 2019.]

OQLF (2018d), invasion brassicole, disponible sur www.granddictionnaire.com/ficheOqlf.aspx?ld_ Fiche=26543641. [Page consultée le 23 février 2019.]

OQLF (2018e), jardin pluvial, disponible sur www.granddictionnaire.com/ficheOqlf.aspx?ld_ Fiche=26511788. [Page consultée le 23 février 2019.]

OQLF (2018f), jeu interactif de foule, disponible sur www.granddictionnaire.com/ficheOqlf.aspx?ld_Fiche=26544116. [Page consultée le 23 février 2019.]

OQLF (2018g), parc inondable, disponible sur www.granddictionnaire.com/ficheOqlf.aspx?ld_ Fiche=26544038. [Page consultée le 23 février 2019.]

OQLF (2018h), place inondable, disponible sur www.granddictionnaire.com/ficheOqlf.aspx?ld_ Fiche=26544049. [Page consultée le 23 février 2019.]

OQLF (2018i), prenez date, disponible sur www.granddictionnaire.com/ficheOqlf.aspx?!d_ Fiche=26543977. [Page consultée le 23 février 2019.]

OQLF (2018j), travailleur plurifonction, disponible sur www.granddictionnaire.com/ficheOqlf.aspx?ld_Fiche=26544127. [Page consultée le 23 février 2019.]

OQLF (2018k), vestiaire d'entrée, disponible sur www.granddictionnaire.com/ficheOqlf.aspx?ld_ Fiche=26544092. [Page consultée le 23 février 2019.]

\section{Sources citées}

Aléong, Stanley, Michel Chrétien, Luc Ostiguy et André Martin (1981), « De la mise en œuvre d’une terminologie de la langue française de l'automobile dans trois écoles d'enseignement technique au Québec : un premier bilan », La Banque des mots, n²1, p. 45-66.

Auger, Pierre (1994), «Implantabilité et acceptabilité terminologiques : les aspects linguistiques d'une socioterminologie du travail », Terminologies nouvelles, n 12, p. 47-57. 
Baillargeon, Stéphane (18 septembre 2017), «L’Office québécois de la langue française applique sa nouvelle politique des emprunts linguistiques », Le Devoir, p. A1 et p. A8.

Bergeron, Marcel (17 décembre 2017), «L'OQLF aux prises avec ses belles-mères », Le Soleil, vol. 121, $n^{\circ} 347$, p. 29.

Bouchard, Chantal (2002), La langue et le nombril : une histoire sociolinguistique du français, Montréal (Québec), Fides.

Bourigault, Didier et Monique Slodzian (1999), « Pour une terminologie textuelle», Terminologies nouvelles, n 19, p. 29-32.

Chrétien, Michel (1981), «Reconnaissance, connaissance et utilisation de la terminologie française, québécoise et anglo-américaine de la haute-fidélité chez le public montréalais », dans JeanMarie Klinkenberg, Danièle Racelle-Latin et Guy Connolly (dir.), Langages et collectivités : Le cas du Québec, Ottawa, Léméac, p. 145-160.

Corbeil, Jean-Claude (2007), « Le rôle de la terminologie en aménagement linguistique : genèse et description de l'approche québécoise », Langages, vol. 168, n 4, p. 92-105.

Corbeil, Jean-Claude et Marie-Éva de Villers (24 septembre 2017), «L'OQLF détourné de sa mission fondamentale», Le Soleil, vol. 121, n² 264, p. 27.

Cormier, Monique C. (27 septembre 2017), «L'OQLF tient compte de l'évolution du Québec », Le Devoir, p. A7.

Diki-Kidiri, Marcel (2007), «Éléments de terminologie culturelle», Cahiers du RIFAL, vol. 26, p. 14-25.

Dupuis, Henriette et André Martin (1985), La féminisation des titres et les leaders d'opinion: une étude exploratoire, Québec, Office de la langue française.

Dutrisac, Robert (21 septembre 2017), « Nouvelle politique linguistique : l'Office québécois de la langue française », Le Devoir, p. A6.

Gambier, Yves (1987), «Problèmes terminologiques des pluies acides: pour une socio-terminologie », Meta : journal des traducteurs, vol. 32, n³, p. 314320.

Heller, Monica, Jean-Paul Bartholomot, Luc Lévy et Luc Ostiguy (1982), Le processus de francisation dans une entreprise montréalaise : une analyse sociolinguistique, Québec: Office de la langue française.

Lafrance, Sylvain et Daniel Gourd (2004), La qualité du français à Radio-Canada : principes directeurs, disponible sur ici.radio-canada.ca/radio/francaismicro/politique.pdf. [Page consultée le 23 février 2019.] 
Maltais, Johanne et Francis Pedneault (2016), « Le traitement de l'emprunt linguistique à l'Office québécois de la langue française », dans Organismes francophones de politique et d'aménagement linguistiques et Office québécois de la langue française (dir.), Les anglicismes : des emprunts à intérêt variable? Recueil des actes: colloque du réseau des Organismes francophones de politique et d'aménagement linguistiques (OPALE), 19 et 20 octobre 2016, Québec : Office québécois de la langue française, p. 277-297.

Maurais, Jacques (7 septembre 2017a), «La démission de l'Office de la langue française », Le Droit.

Maurais, Jacques (4 décembre 2017b), «Anglicismes : L’Office québécois de la langue française ridiculisé », Le Soleil, p. 22.

Office de la langue française (1985), Énoncé d'une politique linguistique relative aux québécismes, Montréal/Québec (Québec), Office de la langue française.

Office québécois de la langue française [OQLF], (5 mars 2004), Politique de l'officialisation linguistique, disponible sur www.oqlf.gouv.qc.ca/ressources/bibliotheque/officialisation/politique_ officialisation_20080425.pdf. [Page consultée le 23 février 2019.]

OQLF (4 septembre 2007), Politique de l'emprunt linguistique, Québec, Office québécois de la langue française.

OQLF (2012a), Le GDT en bref, disponible sur www.granddictionnaire.com/Gdt_en_bref.aspx. [Page consultée le 23 février 2019.]

OQLF (2012b), Nos politiques et nos guides, disponible sur www.granddictionnaire.com/ Politiques_guides.aspx. [Page consultée le 23 février 2019.]

OQLF (2015), Aide du Grand dictionnaire terminologique, disponible sur www.granddictionnaire. com/PDF/Aide_GDT.pdf. [Page consultée le 23 février 2019.]

OQLF (31 janvier 2017), Politique de l'emprunt linguistique, disponible sur www.oqlf.gouv.qc.ca/ ressources/bibliotheque/terminologie/20170330_politique_emprunt.pdf. [Page consultée le 23 février 2019.]

Quirion, Jean (2000), Aspects évaluatifs de l'implantation terminologique, thèse de doctorat, Québec, Université de Montréal.

Quirion, Jean (2003), La mesure de l'implantation terminologique : proposition d'un protocole. Étude terminométrique du domaine des transports au Québec, Québec : Office québécois de la langue française.

Quirion, Jean (2012), « Néologie traductive, néologie aménagiste et néologie collaborative massive: l'unité dans la disparité », Neologica : revue internationale de la néologie, n 6, p. 129-141.

Quirion, Jean (2013), «Facteurs sociaux de la variation terminologique dans les enquêtes d'implantation terminologique : le cas du Québec dans les années suivant l'adoption de la Charte de la langue française », Debate Terminológico, n 9, p. 47-61. 
Quirion, Jean (2014), «La mesure de la variation terminologique comme indice de l'évolution des connaissances dans un environnement bilingue », dans Rita Temmerman et Marc Van Campenhoudt (dir.), Dynamics and Terminology: An interdisciplinary perspective on monolingual and multilingual culture-bound communication, Amsterdam/Philadelphie, John Benjamins, p. 281-302.

René, Nicole (2001), «Implantation terminologique : le rôle de l'Office de la langue française en matière de diffusion terminologique », communication présentée dans le cadre d'une journée de formation, Vitoria, Pays Basque, 13 février.

Taifi, Miloud (2012), «Aménagement linguistique et linguistique de l'usage », Iles D Imesli, n 4, p. 15-24.

Turcotte, Danielle (2013), «Terminologie institutionnelle et usage courant: Créer la continuité pour maximiser l'implantation des propositions », communication présentée à la IX Journée scientifique Realiter [Réseau panlatin de terminologie], Paris, France, 16 octobre 2013.

Usito (6 juin 2017), Accueil, disponible sur www.usito.com. [Page consultée le 23 février 2019.]

Vincent, Nadine (29 septembre 2017), « Ceci n’est pas un "grilled cheese” », Le Devoir, p. A9. 


\section{Annexe}

\section{Légende:}

(p) terme(s) privilégié(s)

(n) terme(s) mentionné(s) au sein d'une note

(c) terme(s) à utiliser dans certains contextes

(v) vedette

\begin{tabular}{|c|c|c|c|c|}
\hline \multirow[t]{3}{*}{ CONCEPT TRAITÉ } & \multirow{3}{*}{$\begin{array}{l}\text { SUGGESTIONS } \\
\text { PRÉALABLES DES } \\
\text { AMÉNAGEURS DU } \\
\text { CTRC }\end{array}$} & \multirow{2}{*}{\multicolumn{2}{|c|}{$\begin{array}{l}\text { SUGGESTIONS DES INTERNAUTES } \\
\text { CLASSÉES DANS L'ORDRE DE LEURS } \\
\text { PRÉFÉRENCES }\end{array}$}} & \multirow{3}{*}{$\begin{array}{l}\text { TERMES ENREGIS- } \\
\text { TRÉS DANS LA FICHE } \\
\text { DU GDT }\end{array}$} \\
\hline & & & & \\
\hline & & Suggestions & $\begin{array}{c}\text { Pourcentage de } \\
\text { votes }\end{array}$ & \\
\hline \multirow[t]{12}{*}{ crowd gaming } & $\mathrm{s} / \mathrm{O}$ & grand jeu interactif & $20 \%$ & $\begin{array}{l}\text { jeu interactif de foule } \\
(p)(v)\end{array}$ \\
\hline & & jeu interactif de foule & $13 \%$ & $\begin{array}{l}\text { jeu coopératif de foule } \\
(p)\end{array}$ \\
\hline & & $\begin{array}{l}\text { jeu d'animation de } \\
\text { foule }\end{array}$ & $10 \%$ & $\begin{array}{l}\text { jeu participatif de foule } \\
(p)\end{array}$ \\
\hline & & jeu de foule & $10 \%$ & \\
\hline & & jeu humain interactif & $10 \%$ & \\
\hline & & jeu participactif & $10 \%$ & \\
\hline & & $\begin{array}{l}\text { jeu participactif de } \\
\text { foule/de masse }\end{array}$ & $10 \%$ & \\
\hline & & jeu de groupe & $7 \%$ & \\
\hline & & $\begin{array}{c}\text { jeu d'animation } \\
\text { interactif de foule/en } \\
\text { masse }\end{array}$ & $3 \%$ & \\
\hline & & jeu de masse & $3 \%$ & \\
\hline & & jeu participatif & $3 \%$ & \\
\hline & & $\begin{array}{l}\text { activité d'animation } \\
\text { interactive de foule/ } \\
\text { en masse }\end{array}$ & $3 \%$ & \\
\hline \multirow[t]{7}{*}{ mudroom } & $\mathrm{s} / \mathrm{O}$ & vestibule à débarras & $10 \%$ & vestiaire d'entrée (p)(v) \\
\hline & & antichambre & $8 \%$ & $\begin{array}{l}\text { vestibule de rangement } \\
\text { (c) }\end{array}$ \\
\hline & & pièce de transition & $8 \%$ & \\
\hline & & tambour-vestiaire & $8 \%$ & \\
\hline & & vestiaire à débarras & $8 \%$ & \\
\hline & & vestibule & $8 \%$ & \\
\hline & & bouerie & $5 \%$ & \\
\hline
\end{tabular}




\begin{tabular}{|c|c|c|c|c|}
\hline \multirow[t]{18}{*}{ CONCEPT TRAITÉ } & \multirow{2}{*}{$\begin{array}{l}\text { SUGGESTIONS } \\
\text { PRÉALABLES DES } \\
\text { AMÉNAGEURS DU } \\
\text { CTRC }\end{array}$} & \multicolumn{2}{|c|}{$\begin{array}{l}\text { SUGGESTIONS DES INTERNAUTES } \\
\text { CLASSÉES DANS L'ORDRE DE LEURS } \\
\text { PRÉFÉRENCES }\end{array}$} & \multirow{2}{*}{$\begin{array}{l}\text { TERMES ENREGIS- } \\
\text { TRÉS DANS LA FICHE } \\
\text { DU GDT }\end{array}$} \\
\hline & & Suggestions & $\begin{array}{c}\text { Pourcentage de } \\
\text { votes }\end{array}$ & \\
\hline & & crasserie & $5 \%$ & \\
\hline & & salle à gadoue & $5 \%$ & \\
\hline & & sas & $5 \%$ & \\
\hline & & sas anti-gadoue & $5 \%$ & \\
\hline & & vestiaire & $5 \%$ & \\
\hline & & $\begin{array}{l}\text { vestibule de } \\
\text { rangement }\end{array}$ & $5 \%$ & \\
\hline & & accès secondaire & $3 \%$ & \\
\hline & & $\begin{array}{c}\text { antichambre de } \\
\text { débarras }\end{array}$ & $3 \%$ & \\
\hline & & $\begin{array}{c}\text { pièce de } \\
\text { débourbage }\end{array}$ & $3 \%$ & \\
\hline & & pièce de décrottage & $3 \%$ & \\
\hline & & salle à boue & $3 \%$ & \\
\hline & & sas anti-boue & $3 \%$ & \\
\hline & & bas-côté & $0 \%$ & \\
\hline & & salle boue & $0 \%$ & \\
\hline & & salle de lavage & $0 \%$ & \\
\hline & & buanderie & $0 \%$ & \\
\hline \multirow[t]{14}{*}{ save the date } & prendre date & à vos agendas! & $17 \%$ & prenez date $(p)(v)$ \\
\hline & prenez date & $\begin{array}{c}\text { réserver/réservez } \\
\text { la date }\end{array}$ & $9 \%$ & réservez la date $(p)$ \\
\hline & & date à réserver & $8 \%$ & réservez cette date $(p)$ \\
\hline & & mémodate & $8 \%$ & date à retenir (c)(n) \\
\hline & & $\begin{array}{l}\text { préinvitation/ } \\
\text { préinviter }\end{array}$ & $8 \%$ & à vos agendas (c)(n) \\
\hline & & $\begin{array}{c}\text { retenir/retenez la } \\
\text { date }\end{array}$ & $8 \%$ & \\
\hline & & $\begin{array}{c}\text { marquez } \\
\text { votre/l'agenda }\end{array}$ & $6 \%$ & \\
\hline & & notez la date & $6 \%$ & \\
\hline & & prenez date & $6 \%$ & \\
\hline & & à mettre à l'agenda & $4 \%$ & \\
\hline & & bloquer la date & $4 \%$ & \\
\hline & & crayonner la date & $4 \%$ & \\
\hline & & faire-partiel & $4 \%$ & \\
\hline & & inscrivez la date & $4 \%$ & \\
\hline
\end{tabular}




\begin{tabular}{|c|c|c|c|c|}
\hline \multirow[t]{9}{*}{ CONCEPT TRAITÉ } & \multirow[t]{2}{*}{$\begin{array}{l}\text { SUGGESTIONS } \\
\text { PRÉALABLES DES } \\
\text { AMÉNAGEURS DU } \\
\text { CTRC }\end{array}$} & \multicolumn{2}{|c|}{$\begin{array}{l}\text { SUGGESTIONS DES INTERNAUTES } \\
\text { CLASSÉES DANS L'ORDRE DE LEURS } \\
\text { PRÉFÉRENCES }\end{array}$} & \multirow[t]{2}{*}{$\begin{array}{l}\text { TERMES ENREGIS- } \\
\text { TRÉS DANS LA FICHE } \\
\text { DU GDT }\end{array}$} \\
\hline & & Suggestions & $\begin{array}{c}\text { Pourcentage de } \\
\text { votes }\end{array}$ & \\
\hline & & invitation & $4 \%$ & \\
\hline & & faire-part & $2 \%$ & \\
\hline & & la date est fixée & $2 \%$ & \\
\hline & & $\begin{array}{l}\text { date tentative (de } \\
\text { rencontre) }\end{array}$ & $0 \%$ & \\
\hline & & demi-faire-part & $0 \%$ & \\
\hline & & $\begin{array}{c}\text { marquer/marquez } \\
\text { la date }\end{array}$ & $0 \%$ & \\
\hline & & $\begin{array}{l}\text { notez-cette-date-à- } \\
\text { votre-agenda }\end{array}$ & $0 \%$ & \\
\hline \multirow[t]{6}{*}{ save-the-date card } & préinvitation & $\begin{array}{c}\text { (carte de) } \\
\text { préinvitation }\end{array}$ & $\mathrm{s} / \mathrm{O}$ & $\begin{array}{l}\text { carte de préinvitation } \\
\text { (p)(v) }\end{array}$ \\
\hline & & carte de date fixée & $\mathrm{s} / \mathrm{O}$ & $\begin{array}{c}\text { carton de préinvitation } \\
\text { (p) }\end{array}$ \\
\hline & & $\begin{array}{l}\text { carte de type } \\
\text { réservation-de-date }\end{array}$ & s/o & préinvitation (p) \\
\hline & & $\begin{array}{c}\text { carte/carton ma- } \\
\text { que-date }\end{array}$ & s/o & \\
\hline & & faire-part & $\mathrm{s} / \mathrm{O}$ & \\
\hline & & invitation & $\mathrm{s} / \mathrm{O}$ & \\
\hline \multirow[t]{13}{*}{ slasheur } & $\mathrm{s} / \mathrm{O}$ & $\begin{array}{l}\text { multiprofessionnel/ } \\
\text { le }\end{array}$ & $11 \%$ & $\begin{array}{l}\text { travailleur plurifonction } \\
\qquad(\mathrm{p})(\mathrm{v})\end{array}$ \\
\hline & & $\begin{array}{c}\text { personne à emplois } \\
\text { multiples }\end{array}$ & $11 \%$ & $\begin{array}{l}\text { travailleuse plurifonc- } \\
\text { tion }(p)\end{array}$ \\
\hline & & $\begin{array}{l}\text { travailleur/se multi- } \\
\text { disciplinaire }\end{array}$ & $11 \%$ & cumulotravailleur (p) \\
\hline & & accumultitre & $9 \%$ & cumulotravailleuse (p) \\
\hline & & $\begin{array}{l}\text { (professionnel/le) } \\
\text { multidisciplinaire }\end{array}$ & $9 \%$ & \\
\hline & & pluriactif/pluriactive & $9 \%$ & \\
\hline & & touche-à-tout & $9 \%$ & \\
\hline & & $\begin{array}{l}\text { sans profession fixe } \\
\text { (SPF) }\end{array}$ & $7 \%$ & \\
\hline & & multiactif/ve & $5 \%$ & \\
\hline & & jongle-titres & $4 \%$ & \\
\hline & & multi-employé(e) & $4 \%$ & \\
\hline & & multipliciste & $4 \%$ & \\
\hline & & $\begin{array}{c}\text { personne à emplois } \\
\text { variés }\end{array}$ & $4 \%$ & \\
\hline
\end{tabular}




\begin{tabular}{|c|c|c|c|c|}
\hline \multirow[t]{6}{*}{ CONCEPT TRAITÉ } & \multirow[t]{2}{*}{$\begin{array}{l}\text { SUGGESTIONS } \\
\text { PRÉALABLES DES } \\
\text { AMÉNAGEURS DU } \\
\text { CTRC }\end{array}$} & \multicolumn{2}{|c|}{$\begin{array}{l}\text { SUGGESTIONS DES INTERNAUTES } \\
\text { CLASSÉES DANS L'ORDRE DE LEURS } \\
\text { PRÉFÉRENCES }\end{array}$} & \multirow[t]{2}{*}{$\begin{array}{l}\text { TERMES ENREGIS- } \\
\text { TRÉS DANS LA FICHE } \\
\text { DU GDT }\end{array}$} \\
\hline & & Suggestions & $\begin{array}{c}\text { Pourcentage de } \\
\text { votes }\end{array}$ & \\
\hline & & étiste & $2 \%$ & \\
\hline & & $\begin{array}{l}\text { (faire du) } \\
\text { multimétier }\end{array}$ & $2 \%$ & \\
\hline & & pluriemployé/e & $2 \%$ & \\
\hline & & $\begin{array}{c}\text { travailleur/se } \\
\text { multi-casquettes }\end{array}$ & $2 \%$ & \\
\hline \multirow[t]{20}{*}{ tap takeover } & prise de fût & $\begin{array}{l}\text { (organiser un) "tout } \\
\text { fût tout flamme" }\end{array}$ & $8 \%$ & $\begin{array}{c}\text { invasion brassicole (p) } \\
(\mathrm{v})\end{array}$ \\
\hline & conquêtes des fûts & $\begin{array}{l}\text { dégustation de } \\
\text { microbrasserie }\end{array}$ & $8 \%$ & incursion brassicole (p) \\
\hline & invasion brassicole & $\begin{array}{l}\text { (organiser un) } \\
\text { "pleins fûts sur..." }\end{array}$ & $8 \%$ & conquête des fûts (p) \\
\hline & incursion brassicole & $\begin{array}{l}\text { quand les microbras- } \\
\text { series mettent la/ } \\
\text { font pression }\end{array}$ & $8 \%$ & \\
\hline & & soirée-dégustation & $8 \%$ & \\
\hline & & $\begin{array}{c}\text { (soirée des) bières } \\
\text { invitées }\end{array}$ & $5 \%$ & \\
\hline & & conquête des fûts & $5 \%$ & \\
\hline & & $\begin{array}{c}\text { conquête des lignes } \\
\text { (de fût) }\end{array}$ & $5 \%$ & \\
\hline & & $\begin{array}{l}\text { (soirée de) fûts en } \\
\text { vedette }\end{array}$ & $5 \%$ & \\
\hline & & incursion brassicole & $5 \%$ & \\
\hline & & invasion brassicole & $5 \%$ & \\
\hline & & mainmise sur les fûts & $5 \%$ & \\
\hline & & $\begin{array}{l}\text { séance de } \\
\text { dégustation }\end{array}$ & $5 \%$ & \\
\hline & & (brasserie) à l'affût & $3 \%$ & \\
\hline & & $\begin{array}{l}\text { appropriation des } \\
\text { fûts }\end{array}$ & $3 \%$ & \\
\hline & & fêtes des brasseurs & $3 \%$ & \\
\hline & & $\begin{array}{l}\text { monopole de } \\
\text { micro(brasserie)s }\end{array}$ & $3 \%$ & \\
\hline & & $\begin{array}{l}\text { monopolisation des } \\
\text { lignes de bière }\end{array}$ & $3 \%$ & \\
\hline & & prise de fût & $3 \%$ & \\
\hline & & $\begin{array}{l}\text { soirée de micro- } \\
\text { monopole }\end{array}$ & $3 \%$ & \\
\hline
\end{tabular}




\begin{tabular}{|c|c|c|c|c|}
\hline \multirow[t]{6}{*}{ CONCEPT TRAITÉ } & \multirow[t]{2}{*}{$\begin{array}{l}\text { SUGGESTIONS } \\
\text { PRÉALABLES DES } \\
\text { AMÉNAGEURS DU } \\
\text { CTRC }\end{array}$} & \multicolumn{2}{|c|}{$\begin{array}{l}\text { SUGGESTIONS DES INTERNAUTES } \\
\text { CLASSÉES DANS L'ORDRE DE LEURS } \\
\text { PRÉFÉRENCES }\end{array}$} & \multirow[t]{2}{*}{$\begin{array}{l}\text { TERMES ENREGIS- } \\
\text { TRÉS DANS LA FICHE } \\
\text { DU GDT }\end{array}$} \\
\hline & & Suggestions & $\begin{array}{c}\text { Pourcentage de } \\
\text { votes }\end{array}$ & \\
\hline & & $\begin{array}{l}\text { soirée micro en } \\
\text { vedette }\end{array}$ & $3 \%$ & \\
\hline & & $\begin{array}{l}\text { dégustation de } \\
\text { bière(s) }\end{array}$ & $0 \%$ & \\
\hline & & $\begin{array}{l}\text { monopolisation des } \\
\text { fûts }\end{array}$ & $0 \%$ & \\
\hline & & $\begin{array}{l}\text { saisie de(s) fûts/des } \\
\text { lignes }\end{array}$ & $0 \%$ & \\
\hline \multirow[t]{18}{*}{ water square } & $\mathrm{s} / \mathrm{O}$ & barc & $\mathrm{s} / \mathrm{O}$ & place inondable $(p)(v)$ \\
\hline & & $\begin{array}{l}\text { bassin de rétention } \\
\text { d'eau de surface } \\
\text { aménagé/ } \\
\text { aménageable }\end{array}$ & $\mathrm{s} / \mathrm{O}$ & $\begin{array}{l}\text { place publique } \\
\text { inondable (p) }\end{array}$ \\
\hline & & & $\mathrm{s} / \mathrm{O}$ & $\begin{array}{c}\text { place multifonctionnelle } \\
\text { inondable }(p)\end{array}$ \\
\hline & & bassin pluvial & $\mathrm{s} / \mathrm{O}$ & $\begin{array}{l}\text { espace public inon- } \\
\text { dable }(p)(v)\end{array}$ \\
\hline & & $\begin{array}{l}\text { bassin pluvial } \\
\text { multiusage }\end{array}$ & $\mathrm{s} / \mathrm{O}$ & jardin pluvial (p)(v) \\
\hline & & $\begin{array}{l}\text { bassin pluvial } \\
\text { polyvalent }\end{array}$ & s/o & jardin de pluie (p) \\
\hline & & bassin sec & $\mathrm{s} / \mathrm{o}$ & jardin inondable (p) \\
\hline & & espace(s) diluvien(s) & $\mathrm{s} / \mathrm{o}$ & parc inondable $(p)(v)$ \\
\hline & & $\begin{array}{l}\text { espace pluvial } \\
\text { (aménagé/ } \\
\text { aménageable) }\end{array}$ & $\mathrm{s} / \mathrm{O}$ & \\
\hline & & esplanade pluviale & $\mathrm{s} / \mathrm{O}$ & \\
\hline & & jardin de pluie & $\mathrm{s} / \mathrm{o}$ & \\
\hline & & parc-bassin & $\mathrm{s} / \mathrm{O}$ & \\
\hline & & parc de pluie & $\mathrm{s} / \mathrm{o}$ & \\
\hline & & parc inondable & $\mathrm{s} / \mathrm{O}$ & \\
\hline & & parc pluvial & $\mathrm{s} / \mathrm{o}$ & \\
\hline & & place d'eau/placed'o & $\mathrm{s} / \mathrm{O}$ & \\
\hline & & $\begin{array}{l}\text { place pluviale } \\
\text { (aménagée/ } \\
\text { aménageable) }\end{array}$ & s/o & \\
\hline & & $\begin{array}{l}\text { zone inondable } \\
\text { récréative (ZIR) }\end{array}$ & s/o & \\
\hline
\end{tabular}




\begin{tabular}{|c|c|c|c|c|}
\hline \multirow[t]{2}{*}{ CONCEPT TRAITÉ } & \multirow[t]{2}{*}{$\begin{array}{l}\text { SUGGESTIONS } \\
\text { PRÉALABLES DES } \\
\text { AMÉNAGEURS DU } \\
\text { CTRC }\end{array}$} & \multicolumn{2}{|c|}{$\begin{array}{l}\text { SUGGESTIONS DES INTERNAUTES } \\
\text { CLASSÉES DANS L'ORDRE DE LEURS } \\
\text { PRÉFÉRENCES }\end{array}$} & \multirow[t]{2}{*}{$\begin{array}{l}\text { TERMES ENREGIS- } \\
\text { TRÉS DANS LA FICHE } \\
\text { DU GDT }\end{array}$} \\
\hline & & Suggestions & $\begin{array}{l}\text { Pourcentage de } \\
\text { votes }\end{array}$ & \\
\hline $\begin{array}{l}\text { zero-acreage } \\
\quad \text { farming }\end{array}$ & & $\begin{array}{l}\text { agriculture ur- } \\
\text { baine (+ indication } \\
\text { du lieu précis [ex. } \\
\text { toit, gouttière, mur]) }\end{array}$ & $30 \%$ & $\begin{array}{l}\text { agriculture urbaine à } \\
\text { surface nulle }(p)(v)\end{array}$ \\
\hline \multirow[t]{8}{*}{ Z-farming } & & $\begin{array}{c}\text { agriculture en bâti } \\
\text { urbain }\end{array}$ & $15 \%$ & \\
\hline & & $\begin{array}{c}\text { culture en bâti } \\
\text { urbain }\end{array}$ & $15 \%$ & \\
\hline & & $\begin{array}{c}\text { ferme sans espace } \\
\text { au sol }\end{array}$ & $15 \%$ & \\
\hline & & $\begin{array}{c}\text { agriculture sans } \\
\text { espace au sol }\end{array}$ & $10 \%$ & \\
\hline & & $\begin{array}{l}\text { agriculture urbaine } \\
\text { en hauteur }\end{array}$ & $5 \%$ & \\
\hline & & $\begin{array}{c}\text { agriculture urbaine } \\
\text { verticale }\end{array}$ & $5 \%$ & \\
\hline & & culture en bâti & $5 \%$ & \\
\hline & & $\begin{array}{c}\text { agriculture sur le bâti } \\
\text { urbain }\end{array}$ & $0 \%$ & \\
\hline
\end{tabular}

